\title{
Emergence of On-Surface Magnetochemistry
}

\author{
Nirmalya Ballav, ${ }^{*}{ }^{\S}$ Christian Wäckerlin, ${ }^{\ddagger}$ Dorota Siewert, ${ }^{\ddagger}$ Peter M. Oppeneer, ${ }^{\dagger}$ and Thomas A. Jung ${ }^{*}, \ddagger$ \\ ${ }^{\S}$ Department of Chemistry, Indian Institute of Science Education and Research (IISER), Pashan, Pune 411 008, India \\ ${ }^{\ddagger}$ Laboratory for Micro and Nanotechnology, Paul Scherrer Institute, 5232 Villigen PSI, Switzerland \\ ${ }^{\dagger}$ Department of Physics and Astronomy, Uppsala University, Box 516, S-751 20 Uppsala, Sweden
}

ABSTRACT: The control of exchange coupling across the molecule-substrate interface is a key feature in molecular spintronics. This Perspective reviews the emerging field of on-surface magnetochemistry, where coordination chemistry is applied to surface-supported metal porphyrins and metal phthalocyanines to control their magnetic properties. The particularities of the surface as a multiatomic ligand or "surface ligand" are introduced. The asymmetry involved in the action of a chemical ligand and a surface ligand on the same planar complexes modifies the well-established "trans effect" to the notion of the

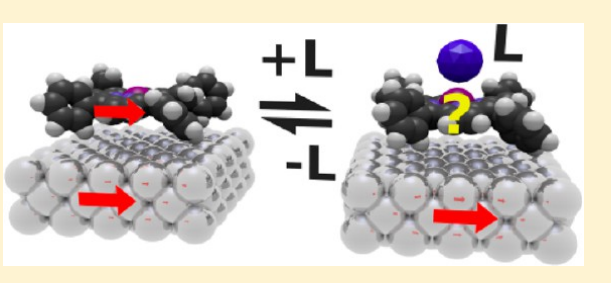
"surface-trans effect". As ad-complexes on ferromagnetic substrates are usually exchange-coupled, the magnetochemical implications of the surface-trans effect are of particular interest. The combined action of the different ligands allows for the reproducible control of spin states in on-surface supramolecular architectures and opens up new ways toward building and operating spin systems at interfaces. Notably, spin-switching has been demonstrated to be controlled collectively via the interaction with a ligand (chemical selectivity) and individually via local addressing at the interface.

\begin{abstract}
A century ago, Alfred Werner laid the foundation of modern coordination chemistry, an achievement for which he was awarded the Nobel Prize in Chemistry in 1913. The innovative concept of primary and secondary valences in coordination complexes that he introduced has since then developed into oxidation states and coordination numbers and become textbook knowledge for introductory chemistry. ${ }^{1}$ Briefly, the transition-metal ion in a given coordination complex with a fixed oxidation state can be either diamagnetic or paramagnetic, depending on the number of the ligands as well as their nature. It is the ligands that are responsible for the splitting of the $d$ orbitals of the metal ion into $t_{2 g}$ and $e_{g}$ levels, which are at the heart of ligand field theory (LFT). ${ }^{1}$ Thereby, ligand coordination directly alters the magnetic properties. Among the existing coordination complexes, particularly spin-bearing four-coordinated square-planar porphyrins and phthalocyanines have received much attention during the last six decades. Here, the coordination number of the metal ion and hence its magnetic properties can be easily modified by coordination with external axial ligands leading to five/six-fold coordination complexes. $^{2}$

Recently, on-surface coordination chemistry of metal porphyrins and metal phthalocyanines has raised increasing attention. $^{3-5}$ In this field of research, coordination chemistry of the planar molecules is studied in the presence of a surface ligand and an optional axial ligand binding to the free on-top site. A central issue is whether the known gas-phase coordination chemistry concepts remain valid for ligand coordination of spin-bearing planar metal-organic molecules assembled on (magnetic) surfaces. Emerging evidence shows that due to the surface-molecule interaction, novel magnetochemical effects arise beyond those known from classical coordination chemistry. Most prominently, the interaction of
\end{abstract}

\section{Emerging evidence shows that due to the surface-molecule interaction, novel magnetochem- ical effects arise beyond those known from classical coordina- tion chemistry.}

spin-polarized electrons in the molecular and surface orbitals can lead to the formation of hybrid molecule-substrate interface states, sometimes called spin-interfaces, that induce magnetic order in paramagnetic molecules up to room temperature and even above. By choice of a suitable top substrate layer, a ferromagnetic (FM) as well as an antiferromagnetic (AFM) coupling between the molecule and the substrate can be engineered. ${ }^{6-15}$ Coordination of these planar metal-organic molecules with the surface ligand has been attributed to the formation of a unique hybrid moleculesubstrate spin interface. Next, the molecule-surface interaction and, via exchange interaction, the molecular spin states can be modified upon coordination to the free ligand position with a molecule like $\mathrm{NO}, \mathrm{NH}_{3}, \mathrm{O}_{2}$, or $\mathrm{CO}$ that could serve the purpose of a chemical stimulus. The binding and release of the axial ligand presents a convenient approach to control the magnetic properties of the individual on-surface complexes. The interrelation between the FM substrate ligand and the axial ligand bound to the on-top site has been referred to as the surface spin-trans effect. ${ }^{16,17}$ This Perspective aims at discussing

Received: May 12, 2013

Accepted: June 26, 2013 
the recent progress made in the field of on-surface magnetochemistry of metal porphyrins and metal phthalocyanines containing first-row transition-metal ions. This emerging field may find its application in molecular spintronics. ${ }^{18}$

Measurement Techniques and Sample Preparation. Spectromicroscopy correlation experiments are performed on atomically well-defined sample surfaces. Figure 1 presents the typical

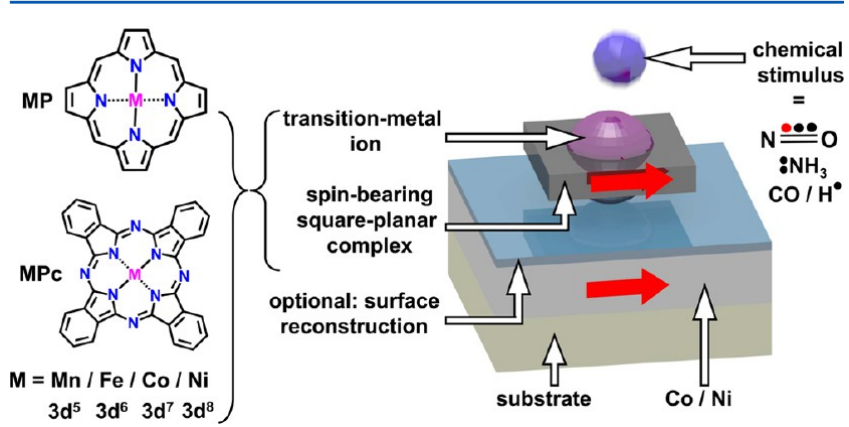

Figure 1. Research design to study the on-surface magnetochemistry of square-planar transition-metal complexes; transition-metal ions (e.g., $\mathrm{Mn}, \mathrm{Fe}, \mathrm{Co}, \mathrm{Ni}$ ) embedded in square-planar porphins (metal-P; MP) or phthalocyanines (metal-Pc; MPc) are studied on FM thin films. Depending on the presence or absence of an optional oxygen reconstruction layer on top of the FM thin film, a ferromagnetically or antiferromagnetically aligned magnetic moment can be observed. This induced magnetic moment in the molecules and its specific response toward axial ligation with a chemical stimulus is then studied by X-ray magnetic circular dichroism (XMCD), spin-polarized scanning tunneling spectroscopy (SP-STS) or spin-resolved (two-photon) photoemission spectroscopy (SR-PS).

research design. All experiments are performed in ultrahigh vacuum (UHV); this environment allows precise dosing of gases acting as optional axial ligands. Standard methods of sample preparation and characterization are the following: ${ }^{10,11}$ transition-metal porphyrins or phthalocyanines are evaporated (slightly less than 1 monolayer) onto freshly prepared $\mathrm{Co}(001)$ or $\mathrm{Ni}(001)$ thin film substrates grown on $\mathrm{Cu}(001)$ kept at room temperature. X-ray photoemission spectroscopy (XPS) and scanning tunneling microscopy (STM) measurements have been combined to estimate the film thickness, the stoichiometry, and the molecular coverage. STM also provides insight into the two-dimensional molecular arrangement and, in general, the morphology of the samples. Chemical stimuli like nitric oxide (NO), ammonia $\left(\mathrm{NH}_{3}\right)$, pyridine, carbon monoxide $(\mathrm{CO})$, and atomic hydrogen $(\mathrm{H})$ are introduced to study on-surface coordination of the metal porphyrins or metal phthalocyanines. To check the reversibility of the chemical spin-tuning events, the samples can be annealed to desorb the axial ligand.

X-ray magnetic circular dichroism (XMCD) spectroscopy provides access to the magnetic properties of both the admolecules and the substrates in an element-specific manner. In this technique, circularly polarized X-rays with opposite helicities (from a synchrotron source) are used to carry out the absorption experiments. For the $3 \mathrm{~d}$ transition metals, the difference of the X-ray absorption (XAS) at the $\mathrm{L}_{2,3}$ edges for opposite helicities provides direct information on the magnetization of both the ad-molecules and the substrates. ${ }^{19}$ Apart from XMCD spectroscopy, several other experimental techniques can be applied to study such molecule-substrate systems. STM has been used recently to study spin-crossover molecules on surfaces ${ }^{20,21}$ and $\mathrm{Cl}$ abstraction from adsorbed tetraphenylporphyrin chloride molecules, inducing a change in magnetic properties. ${ }^{22}$ Spin-polarized scanning tunneling spectroscopy (SP-STS) and microscopy (SP-STM) ${ }^{12,13}$ as well as spin-resolved (one or two-photon) photoemission spectroscopy (SR-PS $)^{15,23}$ were employed to investigate metal phthalocyanine-ferromagnet interface systems. Also, the peak width in XPS allows one to conclude on the presence or absence of unpaired electrons and has been used to study on-surface coordination chemistry on nonmagnetic substrates. ${ }^{4,5,24,25}$

To unveil the origin of the molecule-surface exchange interaction in the presence or absence of axial ligands, numerical modeling based on density functional theory (DFT), supplemented with an additional Coulomb $U$ term $(\mathrm{DFT}+U)$ has been performed. In $\mathrm{DFT}+U$, the strong Coulomb interactions between the $d$ electrons in the open $d$ shell of the metal ion are captured by an additional Hubbard $U$ and exchange constant $J$. This approach has been shown to correctly describe both physisorption and chemisorption of metal-organic molecules ${ }^{26}$ and to provide the correct spin state for a range of effective $U$ values $(=U-J)$, for (i) free molecules and (ii) substrate-adsorbed metal porphyrins and metal phthalocyanines. ${ }^{7,-11}$ In addition, the DFT $+U$ model was used to explain on-surface axial ligation, which was used to control the molecular spin states in various molecule-substrate spin interfaces. ${ }^{17,27}$

On-Surface Magnetochemistry of Planar Molecules on FM Substrates. Control of the molecular spin state and the exchange interaction with the FM substrate by means of either physical or chemical stimuli is a prerequisite for practical applications. Keeping such a notion in mind, the adsorption of first-row transition-metal porphyrins with $\mathrm{d}^{5}, \mathrm{~d}^{6}$, and $\mathrm{d}^{7}$ electronic configurations was investigated systematically. Specifically, this series includes manganese(II)tetraphenylporphyrin (MnTPP), manganese(II)phthalocyanine ( $\mathrm{MnPc})$, iron(II)tetraphenylporphyrin (FeTPP), and cobalt(II)tetraphenylorphyrin (CoTPP) on FM Co or Ni thin films. ${ }^{17}$

The effect of the external ligation with $\mathrm{NO}$ or $\mathrm{NH}_{3}$ onto the hybrid molecule-substrate systems has been studied in order to explore the on-surface magnetochemistry of the $d^{5}-d^{7}$ metal-organic complexes. Figure 2 presents the $\mathrm{Co}, \mathrm{Fe}$, and $\mathrm{Mn} \mathrm{L}_{2,3}$ XAS/XMCD spectra measured before and after axial ligation. ${ }^{17}$ The XAS signal in an on-surface configuration suggests a significant hybridization of electronic states of the molecule and the respective substrate. Consistent with previous reports, FM coupling of all molecular spins to the substrate magnetization can be recognized from the parallel orientations of the $\mathrm{L}_{2,3}$ edges' XMCD signals of Co (from CoTPP), Fe (from FeTPP), and $\mathrm{Mn}$ (from MnTPP or MnPc) with respect to those of the substrate ( $\mathrm{Ni}$ or $\mathrm{Co}$ ). The $\mathrm{FM}$ exchange interaction is reproduced in the DFT $+U$ calculations (Figure $2 \mathrm{a} 3-\mathrm{d} 3$ ) in which the MTPPs were substituted with respective metal porphins (without phenyl rings) to reduce computational efforts. Upon NO exposure, the XAS of the transition-metal ions in the MTPPs was modified (Figure $2 \mathrm{a} 1-\mathrm{cl}$ ), which could be primarily due to the on-surface coordination-induced changes in the oxidation state and the availability of empty states in the $3 \mathrm{~d}$ levels of the ion.

The influence of the surface ligand becomes apparent if one compares on-surface experimental results with the expected modification of the spin states in free complexes, as known from classical coordination chemistry. In bulk/solution, the addition of one axial NO ligand has the following consequences on the spin states: (i) CoTPP $(S=1 / 2 \rightarrow 0)$, (ii) FeTPP $(S=$ 


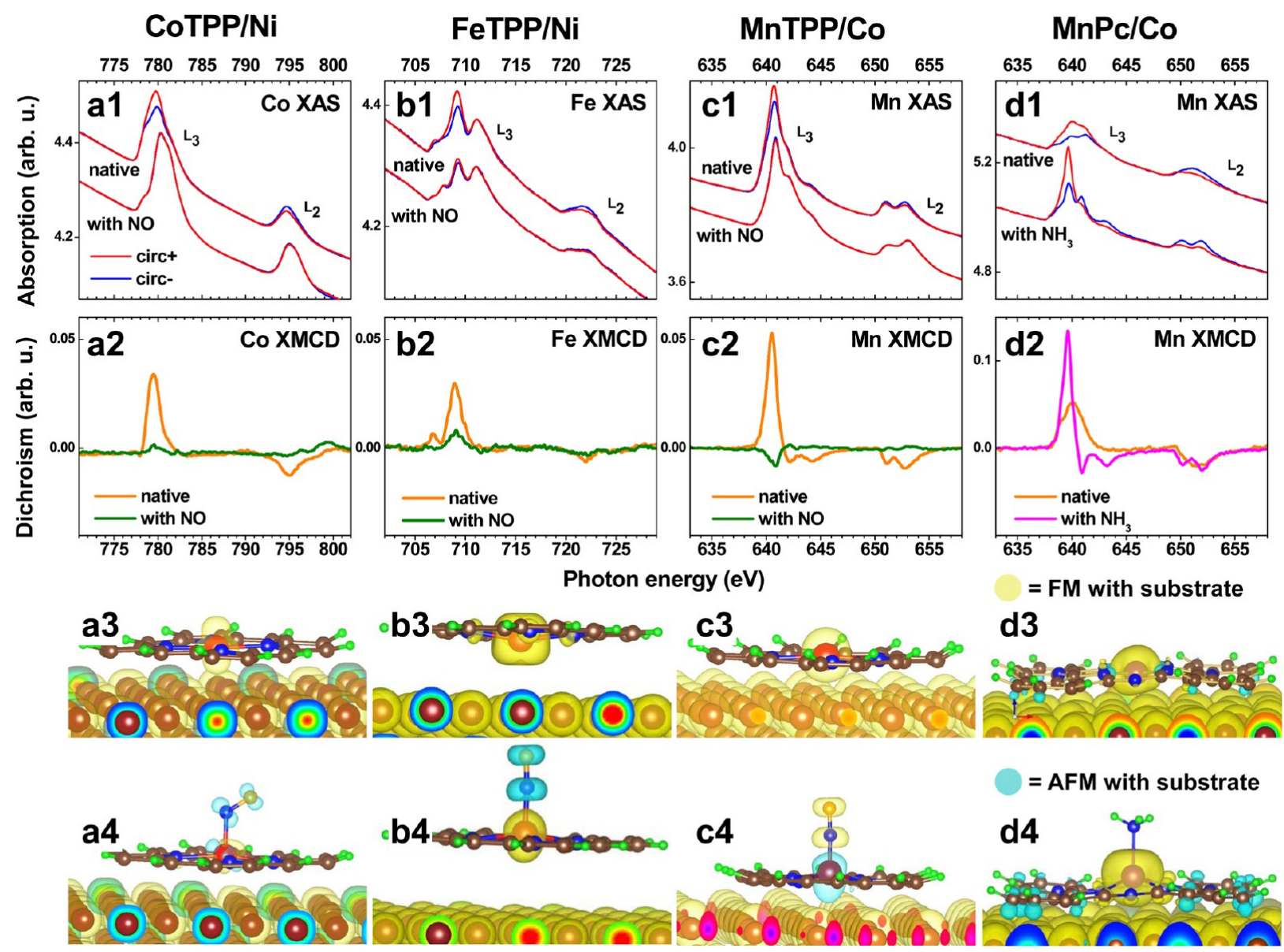

Figure 2. On-surface magnetochemistry of square-planar complexes: XAS of CoTPP/Ni (a1), FeTPP/Ni (b1), MnTPP/Co (c1), and MnPc/Co (d1) measured at the respective L edges, before and after exposure to the gaseous ligand $\left(\mathrm{NO}\right.$ or $\left.\mathrm{NH}_{3}\right)$. The corresponding XMCD spectra (a2-d2) clearly demonstrate an induced magnetic moment in the molecular spin systems. Positive dichroism at the $\mathrm{L}_{3}$ edges corresponds to FM exchange coupling with the substrate. NO ligation modifies the XAS peak shape. The XMCD spectra show the respective magnetic response of the spinbearing molecules; the magnetic moment in CoTPP/Ni is almost completely quenched, for FeTPP/Ni, it is modified and reduced, while for $\mathrm{MnTPP} / \mathrm{Co}$, it is also reduced, but the sign of the XMCD signal is inverted. This corresponds to an antiferromagnetically coupled magnetic moment. In the case of $\mathrm{MnPc} / \mathrm{Co}$ (at low temperatures), the increased circular dichroism after $\mathrm{NH}_{3}$ coordination indicates an increased spin. The magnetization density isosurface plots from the DFT $+U$ calculation of the respective systems before (a3-d3) and after (a4-d4) addition of the ligand illustrate the spin density distribution and the bonding geometry. The light-yellow isosurface denotes spin densities parallel to the substrate (FM), and the light blue isosurface denotes spin densities antiparallel (AFM) to the substrate spin. To reduce the computational effort, the DFT $+U$ calculations were performed on metal porphins instead of MTPPs. Reprinted with permission from ref 17. Copyright 2012, Royal Society of Chemistry, U.K.

$1 \rightarrow 1 / 2$ ), and (iii) MnTPP $(S=5 / 2 \rightarrow 0$ ) (cf. ref 17 and references cited therein). The on-surface coordination with $\mathrm{NO}$ resulted in (i) almost complete quenching of the Co XMCD signal in CoTPP/Ni, (ii) the reduction of Fe XMCD signals, and (iii) the reduction and inversion (from FM to AFM coupling) of the Mn XMCD signals in MnTPP/Co.

While in (i) and (ii) the on-surface result does not strongly deviate from the prediction expected from coordination chemistry, the remaining magnetic moment in case (iii) (MnTPP/Co+NO) clearly demonstrates the impact of the surface ligand on the outcome of the magnetochemical reaction, both concerning the magnetic moment and the parity of the exchange interaction. Considering another ligand, $\mathrm{NH}_{3}$ $(S=0$ ) coordinating to $\mathrm{MnPc} / \mathrm{Co}$ (a strongly chemisorbed system $^{14}$ ) (Figure 2d), the temperature dependency of the Mn XMCD/XAS ratio, before and after exposure to $\mathrm{NH}_{3}$, shows a significant $\mathrm{NH}_{3}$-induced reduction in the molecule-substrate magnetic exchange coupling strength for the $\mathrm{MnPc} / \mathrm{Co}$ system (Figure 3) and an unexpected increase of the $\mathrm{Mn}$ magnetic moment (Figure $2 \mathrm{~d} 2$ ). This is in qualitative agreement with the $\mathrm{DFT}+U$ calculations showing (i) an increase of the magnetic moment from $\sim 3.25$ to $\sim 4.45 \mu_{\mathrm{B}}$, (ii) an increase in the molecule-substrate distance from $\sim 2.30$ to $\sim 2.72 \AA$, and (iii) a significant reduction in the magnetic exchange coupling energy from $\sim 189$ to $\sim 4 \mathrm{meV}$ (cf. also ref 28 , which is concerned with Fe porphyrin on oxygen-reconstructed $\mathrm{Ni}$ ).

Compared to the spin-bearing NO ligand, the role of the diamagnetic $\mathrm{NH}_{3}$ ligand in altering the molecule-substrate spin interaction is remarkable. It has been shown recently by XMCD experiments that the $\mathrm{NH}_{3}$ coordination can even introduce a magnetic moment in a diamagnetic low-spin porphyrin, namely, $\mathrm{Ni}(\mathrm{II}) \mathrm{TPP}$, on a Co surface. ${ }^{27}$ From $\mathrm{DFT}+U$ calculations, it was found for the $\mathrm{NiP} / \mathrm{Co}$ system that the $\mathrm{NiP}$ molecule was in an $S \approx 0$ state, with the $3 \mathrm{~d}_{z^{2}}$ orbital doubly occupied and the $\mathrm{d}_{x^{2}-y^{2}}$ orbital empty. Upon axial ligation with $\mathrm{NH}_{3}$, the orbital occupation is computed to change to singly occupied $\mathrm{d}_{z}{ }^{2}$ and $\mathrm{d}_{x^{2}-y^{2}}$ orbitals, rendering a triplet spin $S \approx 1$ (magnetic moment of $\sim 1.91 \mu_{\mathrm{B}}$ ), which in addition was FM coupled to the Co 


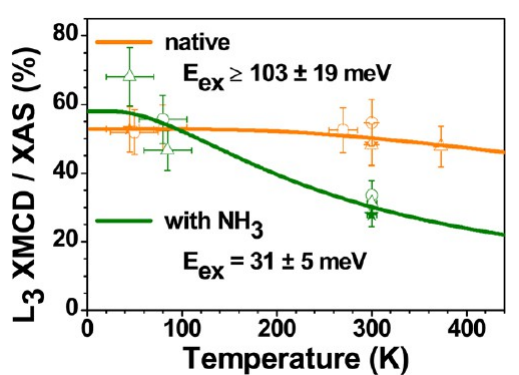

Figure 3. Temperature dependence of the $\mathrm{XMCD} / \mathrm{XAS}$ ratio of $\mathrm{MnPc} / \mathrm{Co}$ before and after $\mathrm{NH}_{3}$ exposure. The symbols (e.g., triangles, etc.) mark different experimental runs, and $E_{\mathrm{ex}}$ is the energy of the magnetic exchange coupling as determined experimentally by fitting the experimental data with Brillouin functions (solid lines). The data provide evidence for an influence of the axial ligand onto the molecule-substrate exchange interaction strength. XMCD/XAS ratios of native and $\mathrm{NH}_{3}$-dosed $\mathrm{MnPc} / \mathrm{Co}$ are shown. In the case of native $\mathrm{MnPc} / \mathrm{Co}$, the $\mathrm{XMCD} / \mathrm{XAS}$ ratio varies only very slightly with temperature, evidencing a very strong exchange interaction. Upon $\mathrm{NH}_{3}$ ligation, the XMCD/XAS ratio at $\sim 300 \mathrm{~K}$ is significantly lower compared to that of the low-temperature case. This evidences a significantly reduced exchange coupling strength upon $\mathrm{NH}_{3}$ ligation. Reprinted with permission from ref 17. Copyright 2012, Royal Society of Chemistry, U.K.

substrate. Interestingly, the $\mathrm{Ni}-\mathrm{Co}$ distance for the $\mathrm{NiP} / \mathrm{Co}$ $(\sim 3.09 \AA)$ system was significantly lower than the value obtained for the $\mathrm{NH}_{3}-\mathrm{NiP} / \mathrm{Co}(\sim 3.59 \AA)$ system.

Despite several distinctive magnetic properties manifesting as a result of the on-surface coordination of $\mathrm{NO}$ or $\mathrm{NH}_{3}$ ligands to metal porphyrins or metal phthalocyanine, one aspect was consistently obtained for all investigated systems, which is the increase of the molecule-substrate distance upon external axial ligation. This corresponds to a weakening of the moleculesubstrate bond. Hence, the combined experimental and theoretical data provided evidence that on-surface axial ligation leads to a structural trans effect, which not only affects the molecular spin state but also can influence the sign and strength of the magnetic exchange interaction, that is, a surface spintrans effect. On account of the weakening of the chemical bond (increased bond length) with the surface, as regularly observed in various ad-complex-substrate systems, in general, a reduced magnetic exchange interaction should be expected. However, the modified spin state and orbital occupation after ligation as well as the orbital energies with respect to the band structure of the FM substrate might play a decisive role that can lead to surprising effects. Such an influence of on-surface coordination on the molecule-substrate magnetic exchange interactions is as yet largely unexplored.

\section{On-surface axial ligation leads to a structural trans effect, which not only affects the molecular spin state but also can influence the sign and strength of the magnetic exchange interaction.}

AFM Coupling and Molecular Self-Assembly. So far, we have discussed the usual FM coupling in native molecule-substrate spin interfaces, with and without on-surface coordination of an external axial ligand. Looking at transition-metal monoxides, an
AFM coupling within pairs of metal ion spins is well-known and can be understood by a superexchange interaction mechanism via the oxygen atom. ${ }^{19}$ Engineering of the same kind of antiparallel interaction is, in principle, possible if one can put an oxygen spacer between the molecule and substrate. Indeed, as the first example, an AFM coupling (from XMCD measurements; see Figure 4) in a molecule-substrate interface

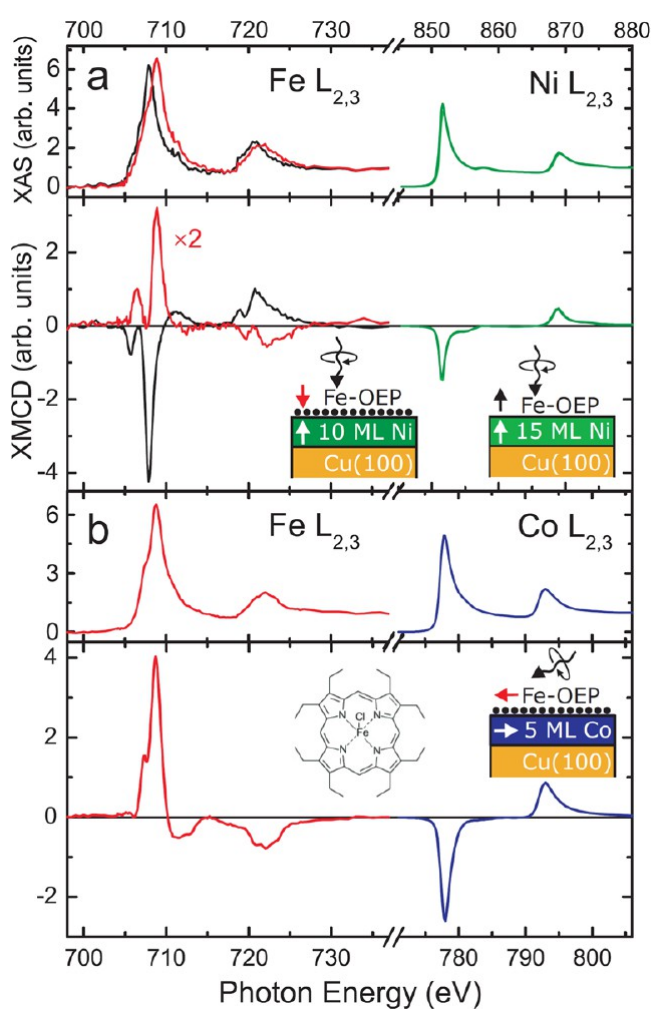

Figure 4. $\mathrm{Fe} \mathrm{L}_{2,3} \mathrm{XAS}$ and XMCD spectra of $0.6 \mathrm{ML} \mathrm{Fe}-\mathrm{OEP}$ on $\mathrm{O} / \mathrm{Ni}$ (a) and $\mathrm{O} / \mathrm{Co}$ (b) (full red or gray lines) and on $\mathrm{Ni}$ (dashed black lines, panel (a)). The corresponding $\mathrm{Ni}$ and Co spectra are shown on the right. (Insets) Sketches of the corresponding samples and of the Fe porphyrin molecule. Reprinted with permission from ref 9. Copyright 2009, American Physical Society, U.S.A.

was demonstrated by decorating the thin film $\mathrm{Co}$ or $\mathrm{Ni}$ substrate with oxygen atoms followed by the deposition of spinbearing metal porphyrin, namely, $\mathrm{Fe}(\mathrm{II})$ octaethylporphyrin chloride $(\mathrm{FeOEPCl})$, that is, $\mathrm{FeOEPCl} / \mathrm{O} / \mathrm{Co}$ or $\mathrm{FeOEPCl} /$ $\mathrm{O} / \mathrm{Ni}^{9}{ }^{9}$ From $\mathrm{DFT}+U$ calculations, the observed antiparallel spin alignment between the molecule and substrate was explained by means of $\mathrm{Fe}-\mathrm{O}-\mathrm{Co}$ or $\mathrm{Fe}-\mathrm{O}-\mathrm{Ni}$ superexchange interactions. Similarly, a $\mathrm{MnTPPCl} / \mathrm{O} / \mathrm{Co}$ system was fabricated, and an AFM coupling could be detected from the antiparallel alignment of the $\mathrm{Mn}$ and Co L edges' XMCD signals. ${ }^{10}$ An important issue that initially remained unresolved was the difference in the molecular assembly of spin-bearing metal porphyrins or metal phthalocyanines on metallic versus oxygen-covered FM thin film substrates $(\mathrm{O} / \mathrm{Co}$ or $\mathrm{O} / \mathrm{Ni})$.

STM data presented in Figure 5 revealed remarkable differences in the morphologies of the metallic Co and O/Co substrates. The LEED pattern showed a $c(2 \times 2)$ oxygen superstructure, as reported earlier in the case of surfactantmediated growth of metallic thin films. Furthermore, the molecular assemblies of $\mathrm{MnTPPCl}$ onto those substrates were distinctively different; $\mathrm{MnTPPCl}$ molecules were found to 

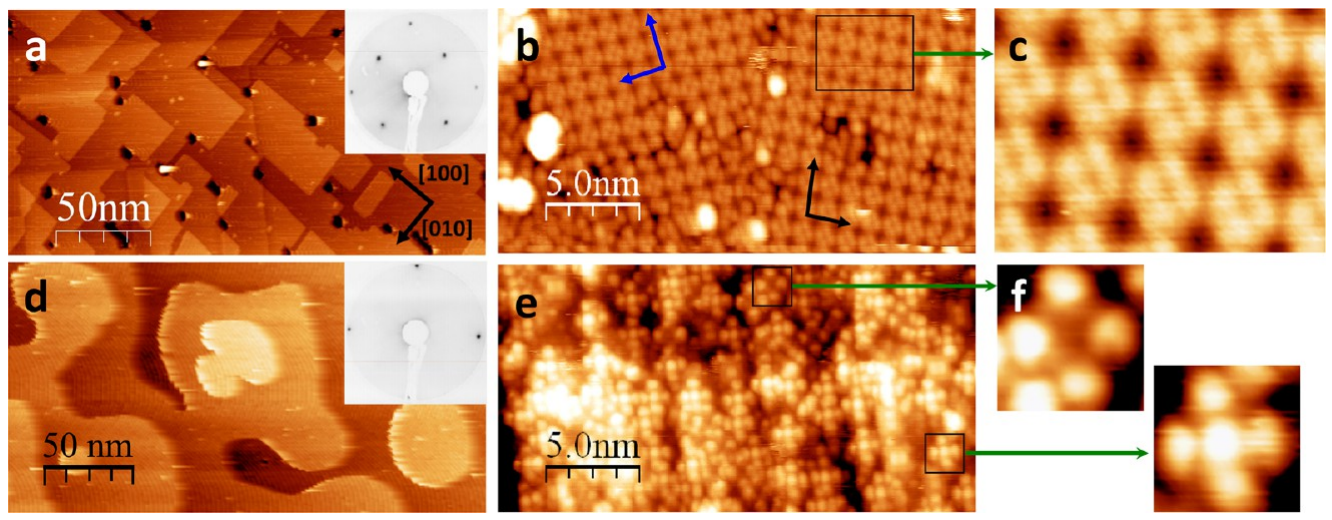

Figure 5. STM images: (a,b) O/Co(001) without and with $\mathrm{MnTPPCl}$ molecules, respectively. The black arrows in (a) represent in-plane crystallographic directions that apply to all STM images; the blue and black arrows in (b) indicate two mirror domains also observed in LEED (see ref 10). (d,e) $\mathrm{Co}(001)$ without and with $\mathrm{MnTPPCl}$ molecules, respectively. (c) Zoom into self-assembled molecules on $\mathrm{O} / \mathrm{Co}(001)$ and (f) zoom into two types of molecules on Co(001). (Insets) LEED pattern taken (a) at $53.2 \mathrm{eV}$ on O/Co(001) and (d) at $53.2 \mathrm{eV}$ on $\mathrm{Co}(001)$. Acquisition parameters for the STM images $(I, V)$ : (a) $0.26 \mathrm{nA},-1.5 \mathrm{~V}$; (b) $0.08 \mathrm{nA},-3.0 \mathrm{~V}$; (d) $0.05 \mathrm{nA},-1.7 \mathrm{~V}$; and (e) $0.12 \mathrm{nA},-2.3 \mathrm{~V}$. Reprinted with permission from ref 10. Copyright 2010, American Chemical Society, U.S.A.

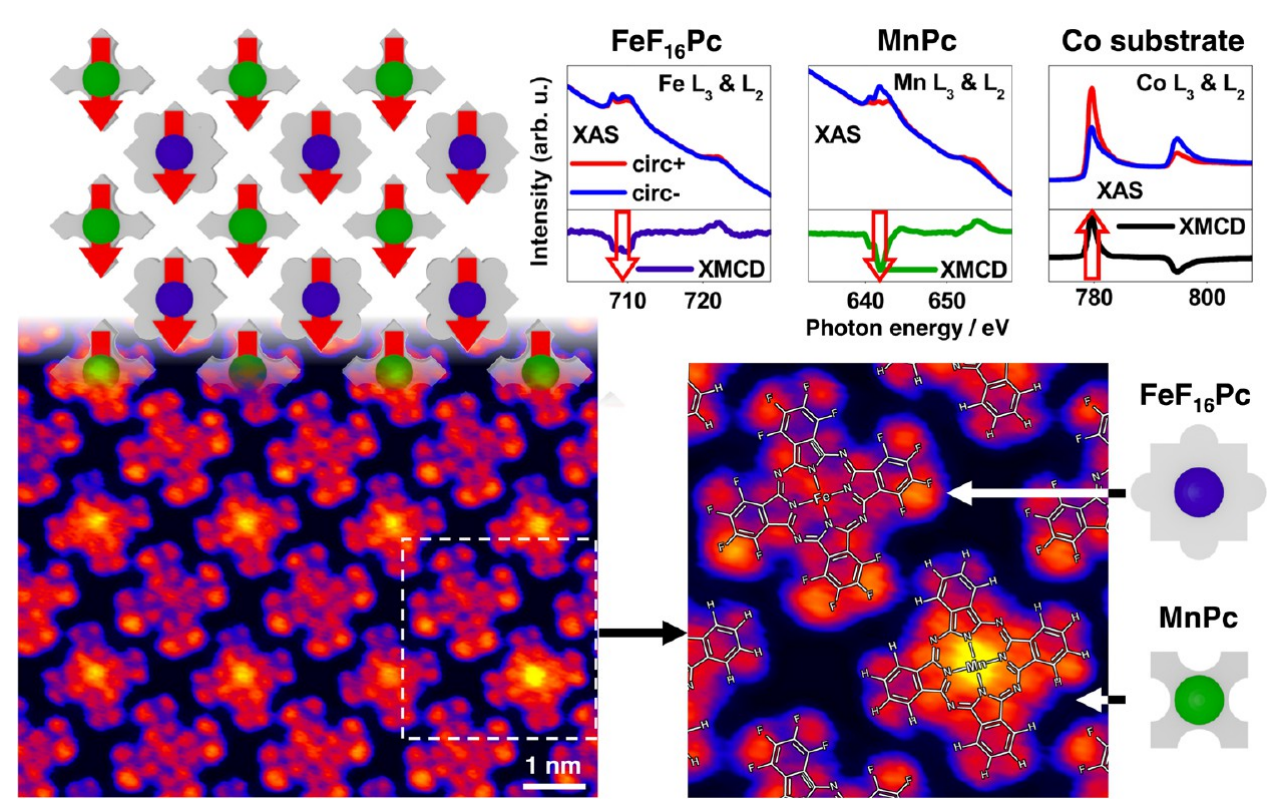

Figure 6. Chemical structures of $\mathrm{FeF}_{16} \mathrm{Pc}$ and $\mathrm{MnPc}$ are superimposed on the STM image, which directly shows the supramolecular chessboard-like 2D lattice and the intramolecular electronic structure. Thus, the resulting molecular array consists of two superimposed spin-bearing lattices, $\mathrm{Fe}$ (dark-blue circles in jigsaw pieces) and Mn (green circles in jigsaw pieces). XAS and XMCD on the respective $\mathrm{L}_{3,2}$ edges identify the antiparallel orientation of the Fe or Mn magnetic moments in the self-assembled 2D array with respect to the magnetization of the oxygen-covered FM Co substrate. This antiparallel alignment stems from the superexchange interaction via the oxygen surface reconstruction. The data shown here are obtained at $\sim 70 \mathrm{~K}$, but the spin alignment as well as the supramolecular arrangement is strong enough to be observed up to room temperature. Reprinted with permission from ref 31. Copyright 2013, Wiley-VCH Verlag GmbH \& Co. KGaA, Germany.

adsorb in a random fashion onto Co surface, whereas on $\mathrm{O} /$ $\mathrm{Co}(001)$, ordered and self-assembled molecular domains were observed (cf. Figure 5). The occurrence of diffusion, as visible by the spontaneous molecular self-assembly of $\mathrm{MnTPPCl}$ on the $\mathrm{O} / \mathrm{Co}$ substrate, suggests a weakened molecule-substrate electronic interaction. Thus, in general, spectromicroscopy correlation along with numerical modeling is very much essential in unraveling the complex molecule-substrate interaction. This observation of molecular self-assembly of metal porphyrins on oxygen-covered FM thin film substrates paved the way toward building chemically tunable supramolecular spin interface structures (vide infra).

Chemically Programmable 2D Supramolecular Spin Arrays. Spin-bearing ad-atoms can be arranged in a specific manner on a surface by atom-positioning techniques, and moreover, those atoms can be individually probed by STM/STS. ${ }^{29,30}$ However, this piece-by-piece assembly of the atoms is a very timeconsuming process and is inherently difficult to scale up toward larger functional structures. Also, the current lithographic technology does not allow for atomic precision. As an alternative, it has been shown that a simple $2 \mathrm{D}$ magnetic structure can be built by molecular self-assembly. A supramolecular chessboard-like structure, consisting of two-different spin centers, was formed by mere co-evaporation of the functionalized building blocks. Specifically, the self-assembly of $\mathrm{MnPc}$ and perfluorinated iron(II)phthalocyanine $\left(\mathrm{FeF}_{16} \mathrm{Pc}\right)$ on the $\mathrm{O} / \mathrm{Co}$ substrate resulted in the formation of a bimolecular chessboard-like pattern (shown in Figure 6) via intermolecular 


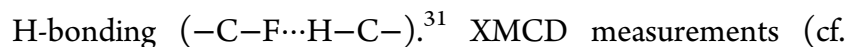
Figure 6) clearly showed an AFM coupling of both $\mathrm{Mn}$ and $\mathrm{Fe}$ centers to the Co substrate. The molecular spin states in this 2D supramolecular layer can be reversibly manipulated by the on-surface coordination with an external $\mathrm{NH}_{3}$ ligand. Distinctive responses of $\mathrm{NH}_{3}$ ligation to $\mathrm{MnPc}$ and $\mathrm{FeF}_{16} \mathrm{Pc}$ allowed selective control of the molecular spin states of the $\mathrm{Mn}$ or Fe ions. The coordination of $\mathrm{NH}_{3}$ via its lone pair modified the ligand field of the $\mathrm{Fe}$ ion in such way that it yielded a lowspin configuration ( $S=0$, quenching of the Fe-XMCD signal was observed), while for the $\mathrm{MnPc}$, the $\mathrm{NH}_{3}$ ligation did not quench the spin but could merely modify it (different $\mathrm{Mn}$ XMCD peak shape compared to the native system). Lowtemperature STM $(\sim 78 \mathrm{~K})$ experiments enabled visualization of the coordination of $\mathrm{NH}_{3}$ to both $\mathrm{MnPc}$ and $\mathrm{FeF}_{16} \mathrm{Pc}$ molecules. Interestingly, upon increasing the sample temperature to $\sim 130 \mathrm{~K}$, the $\mathrm{NH}_{3}$ ligands were only found on the $\mathrm{MnPc}$ molecules, which suggests a relatively higher affinity of $\mathrm{NH}_{3}$ to $\mathrm{MnPc}$, in comparison to $\mathrm{FeF}_{16} \mathrm{Pc}$. Overall, the power of molecular self-assembly can be combined with on-surface coordination chemistry, and thereby, we can explore and exploit the fabrication of novel chemically addressable $2 \mathrm{D}$ supramolecular spin-arrays.

\section{Molecular self-assembly can be combined with on-surface coor- dination chemistry to thereby explore and exploit the fabrica- tion of novel chemically address- able 2D supramolecular spin arrays.}

On-Surface Metal Organic Coordination Oligomers and Polymers. Metal-organic frameworks (MOFs) or coordination polymers can provide systems with unprecedented configurability that, in addition, allow for the tuning of magnetic properties in $3 \mathrm{D}$ as well as in surface-supported $2 \mathrm{D}$ networks. The structures of 2D MOFs and, for example, their electronic and magnetic properties can be readily tuned by selecting suitable combinations of metal ion(s) and ligand(s). A remarkable example of on-surface magnetochemistry has been realized by Gambardella et al.; an on-surface array of magnetic coordination tetramers, for example, has been fabricated on a $\mathrm{Cu}$ surface from Fe ions and terephthalic acid (TPA). ${ }^{32}$ Each $\mathrm{Fe}$ ion was found to coordinate with four TPA ligands via carboxylate bonds forming a tetramer $\mathrm{Fe}(\mathrm{TPA})_{4}$ held in $2 \mathrm{D}$ arrays by weak H-bonds. The coordination sphere of the Fe ion is not fully saturated in the $\mathrm{Fe}(\mathrm{TPA})_{4}$ tetramer, thereby offering active sites for further coordination. XMCD has been used to investigate the magnetic properties of this coordination tetramer before and after reaction with $\mathrm{O}_{2}$ acting as an external axial ligand. A strong magnetic anisotropy behavior of the $\mathrm{Fe}$ ions was observed; Figure 7 clearly shows that the easy magnetization axis of native $\mathrm{Fe}(\mathrm{TPA})_{4}$ lies in-plane. $\mathrm{O}_{2}$ coordination leads to reorientation of the easy magnetization axis to the out-of-plane direction. These experiments have been performed at high magnetic fields on nonmagnetic samples, thereby measuring the intrinsic properties of a single layer of the on-surface complex.

In the context of fully developed spin-bearing MOFs on surfaces, it is worth mentioning that a FM coupling between Fe centers has been recently demonstrated in a planar coordination polymer consisting of $\mathrm{Fe}$ ions and tris(4pyridyl)triazene (T4TP) ligands. ${ }^{33}$ Thus, on-surface coordination complexes provide a very important configurable material to tune spin-bearing layers employing on-surface magnetochemistry.
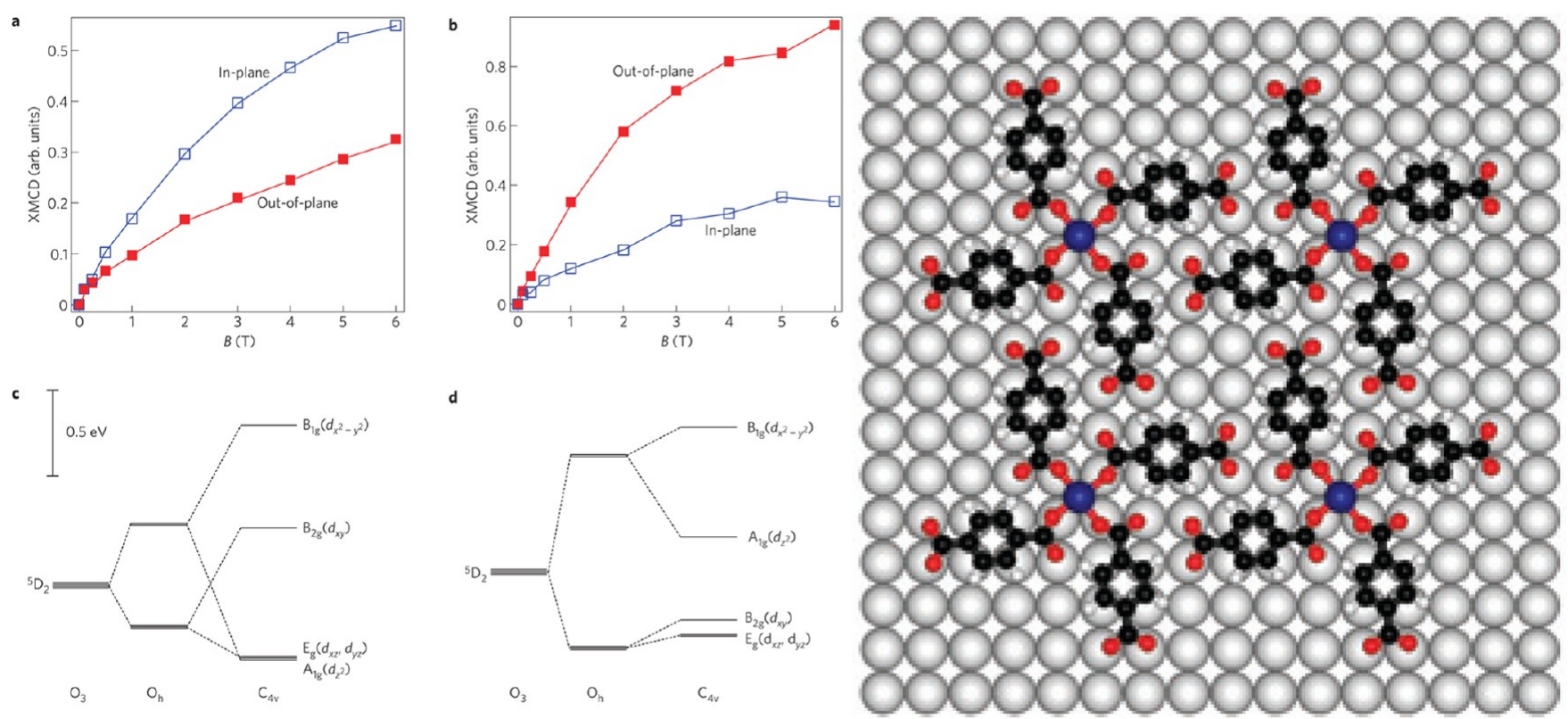

Figure 7. (Left) $\mathrm{Fe}(\mathrm{TPA})_{4}$ (a) and $\mathrm{O}_{2}-\mathrm{Fe}(\mathrm{TPA})_{4}$ (b) magnetization measured at $T=8 \mathrm{~K}$ with the field applied out-of-plane $\left(\theta=0^{\circ}\right.$, filled symbols) and close to the in-plane direction $\left(\theta=70^{\circ}\right.$, open symbols). The data points represent the Fe XMCD intensity integrated over the $\mathrm{L}_{3}$ edge and normalized by the corresponding $\mathrm{L}_{3}$-integrated XAS intensity. Crystal field diagrams of $\mathrm{Fe}(\mathrm{TPA})_{4}$ (c) and $\mathrm{O}_{2}-\mathrm{Fe}(\mathrm{TPA})_{4}$ (d) derived from the XAS multiplet simulations. (Right) Top-view model of $\mathrm{Fe}(\mathrm{TPA})_{4}$ coordination and the supporting $\mathrm{Cu}(100)$ substrate. Reprinted with permission from ref 32. Copyright 2009, Nature Publishing Group, U.K. 
Single-Molecule Spin-Switching. In view of the impressive capabilities to ligate magnetically functional layers via axial ligands, the expectation has been raised that also individual molecular spin-switching events can be realized and detected by local probes. Very recently, such events have been realized by manipulation of $\mathrm{CO}$ ligands on $\mathrm{MnPc}$ molecules adsorbed on $\mathrm{Bi}^{34}$ Notably, here, the spin state of MnPc changes from $S=3$ / 2 to 1 upon adsorption on the Bi surface (cf. Figure 8 ). It is
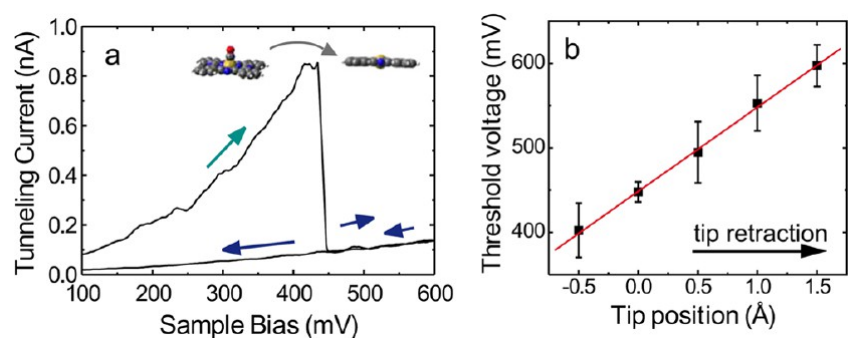

C
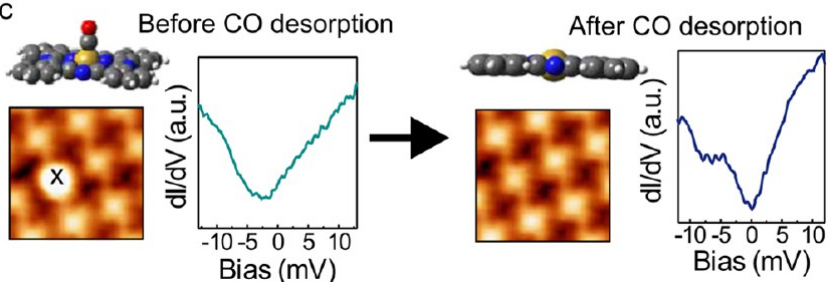

Figure 8. On-surface magnetochemistry on an individual molecule: STM-induced desorption of $\mathrm{CO}$ from MnPc. (a) $I-V$ characteristics recorded during the tip-induced desorption experiment. A sudden drop in the current marks the threshold voltage for CO detachment. (b) Threshold voltage plot versus tip-sample distance. The linear fit of the data indicates that the process is driven by a threshold electric field of $1 \mathrm{~V} / \mathrm{nm}$. (c) STM image and $\mathrm{d} I / \mathrm{d} V$ spectrum of the molecule before and after $\mathrm{CO}$ desorption. Reprinted with permission from ref 34. Copyright 2012, American Physical Society, U.S.A.

reported that upon CO ligation, the spin state of the $\mathrm{Mn}$ ion on $\mathrm{Bi}$ is switched from the $S=1$ to $1 / 2$ spin state. After desorption of individual CO ligands excited locally on one molecule by the STM tip, the initial state is recovered. Note that the spin states of the individual complexes were detected by observing the characteristic zero-bias anomaly (ZBA) in STM. It has also been shown that the modification of the ligand field by sitespecific charge transfer by Li doping can affect the magnetic properties of $\mathrm{CuPc}^{35}$ In another report, the spin state in $\mathrm{MnPc}$ molecules adsorbed on a $\mathrm{Au}$ surface was reversibly switched between $S=3 / 2$ and 1 by on-surface coordination of hydrogen atoms to the $\mathrm{Mn}$ complexes. ${ }^{36}$ Note that even a single $\mathrm{H}$-atom has been successfully used to ligate and tune magnetism at the organic/inorganic interface; possibly this provides the spatial size limit for a "chemical switch".

Outlook. The fundamental properties of organic spin-bearing materials and layers and their control via on-surface magnetochemistry is a rapidly emerging field. In only a few years since the first report of an exceptionally strong exchange interaction that induces a magnetic moment in initial paramagnetic surface ad-molecules, a large portfolio of proof-of-principle experiments has been reported. A wide range of chemical stimuli, both spinbearing and non-spin-bearing, have been used to tune or switch the magnetic moment, the exchange interaction between the magnetic moments of the molecule and the substrate, and the magnetic anisotropy of specific on-surface complexes. The analysis of this broad portfolio of reports with numerical DFT calculations has provided deep insight into the chemical adcomplex substrate interactions and their consequences for the control and tuning of the magnetic interaction. Furthermore, the role of the substrate, via the surface termination influencing the exchange interaction and the assembly, has been studied. In combination with a supramolecular chemistry approach, periodic arrays of bimolecular spin systems have been fabricated. The individual response of the two different spin centers toward axial ligation has been employed to selectively control the magnetization in these engineered and spinfunctional supramolecular layers. While at current the predominant result puts the focus on porphyrins and phthalocyanines, it is important to highlight that coordination oligomers and MOFs offer an even wider range of tunability involving complex engineered spin systems. Recently, singlemolecule experiments using local probe experiments have been showcasing the capability to reversibly operate single-spin systems by on-surface magnetochemistry and single-molecule manipulation.

On the basis of the current state of the art, there exists a considerable opportunity for fundamental breakthroughs and demonstrations of reversible and device-relevant switching behavior down to the single-molecule or the single-spin level. In particular, we would like to mention the following: (i) the systematic exploration of molecule-substrate spin interfaces where the substrate could be an AFM, topological insulator, or superconducting type; (ii) the use of physical stimuli like temperature or light to control the molecular spin states, for example, via conformational factors programmed into the molecule; and (iii) the fabrication of spin-bearing supramolecular surface structures in which different magnetic centers are exchange-coupled.

\section{There exists a considerable op- portunity for fundamental break- throughs and demonstrations of reversible and device-relevant switching behavior down to the single-molecule or the single-spin level.}

As for further experimental progress, a number of opportunities are envisioned to benefit from the implementation of pump-probe techniques to access the ultrafast electron and spin dynamics in well-architectured molecule-substrate interfaces. On these grounds, it can be foreseen that on-surface magnetochemistry will continue to develop rapidly. This trend is further supported by the projections that organic spintronics and their applications may undergo a similar gain in importance, comparable to what has been observed for inorganic spintronics during the last years.

\section{AUTHOR INFORMATION}

\section{Corresponding Author}

*E-mail: nballav@iiserpune.ac.in (N.B.); thomas.jung@psi.ch (T.A.J.).

\section{Author Contributions}

All authors have contributed in compiling the draft and have given approval for the final version of the manuscript. 


\section{Notes}

The authors declare no competing financial interest.

\section{Biographies}

Nirmalya Ballav is an assistant Professor of Chemistry at the Indian Institute of Science Education and Research (IISER), Pune, India. His research focuses on spin-bearing molecule-substrate interfaces, molecular spin-switching, molecular self-assembly, electron beam chemical lithography, graphene, nanoparticles, and polymer-based organic-inorganic hybrid nanocomposite materials. Website link: http://www.iiserpune.ac.in/ nballav

Christian Wäckerlin received his B.Sc. and M.Sc. in Nanoscience from the University of Basel. He performed his Ph.D. work at the Paul Scherrer Institute and obtained the degree from the University of Basel in 2013. In his Ph.D., he studied on-surface chemistry and, in particular, the on-surface magnetochemistry of square-planar complexes. Website link: http://christian.waeckerlin.com

Dorota Siewert received her B.Sc. and M.Sc. in solid-states physics from the AGH University of Science and Technology, Krakow. She performed her Ph.D. work at the Paul Scherrer Institute and obtained her degree in Physics from the University of Basel in 2011. In her Ph.D., she studied surface-supported metal-organic systems and onsurface magnetism.

Peter M. Oppeneer is Professor in physics at Uppsala University, Sweden. His research focuses on computational-theoretical materials science, in particular, chemical and magnetic processes occurring at molecule-substrate interfaces, the theory of spin-switching induced in metal-organic molecules and spin-crossover materials, and magnetochemistry of spin-bearing complexes. Website link: http://www. physics.uu.se/en/page/peter-oppeneer

Thomas A. Jung leads the Molecular Nanoscience group at the Paul Scherrer Institute (ETH domain) and holds a Professorship in Nanoscience at the University of Basel, Switzerland. He is interested in new concepts for functional devices on the atomic and molecular scales, which refers to understanding mechanic, electronic, spintronic, and optic properties of architectured nanometer-sized units at contacting interfaces. Website links: http://www.physik.unibas.ch/ $\mathrm{dept/pages/en/personnel/jung.htm} \mathrm{and} \mathrm{http://www.psi.ch/lmn/}$ molecular-nanoscience

\section{ACKNOWLEDGMENTS}

Financial support from the National Centre of Competence in Research Nanosciences, Swiss Nanoscience Institute, Swiss National Science Foundation, and Holcim Foundation for the Advancement of Scientific Research, Switzerland; from the Swedish-Indian Research Links Programme, Sweden; and from the Indian Institute of Science Education and Research (IISER) Pune, India are acknowledged. The authors sincerely thank Kartick Tarafder, Ehesan Ali, Kathrin Müller, Cristian Iacovita, Jan Girovsky, Jan Nowakowski, Aneliia Shchyrba, Armin Kleibert, Frithjof Nolting, Shi-Xia Liu, Silvio Decurtins, and Rolf Schelldorfer for their involvement in various parts of the reported research. The authors thank Simon Wenner for the animated 3D graphics used in the video of this Perspective. This Perspective is dedicated to Prof. K. N. Ganesh (IISER Pune) on the occasion of his 60th birthday.

\section{REFERENCES}

(1) Huheey, J. Inorganic Chemistry: Principles of Structure and Reactivity, 4th ed.; Harper Collins College Publishers: New York, 1993.

(2) Kadish, K. M.; Smith, K. M.; Guilard, R. The Porphyrin Handbook; Academic Press: St. Louis, MO, 1999.
(3) Lukasczyk, T.; Flechtner, K.; Merte, L. R.; Jux, N.; Maier, F.; Gottfried, J. M.; Steinrück, H.-P. Interaction of Cobalt(II) Tetraarylporphyrins with a $\mathrm{Ag}(111)$ Surface Studied with Photoelectron Spectroscopy. J. Phys. Chem. C 2007, 111, 3090-3098.

(4) Flechtner, K.; Kretschmann, A.; Steinrück, H.-P.; Gottfried, J. M. NO-Induced Reversible Switching of the Electronic Interaction between a Porphyrin-Coordinated Cobalt Ion and a Silver Surface. J. Am. Chem. Soc. 2007, 129, 12110-12111.

(5) Hieringer, W.; Flechtner, K.; Kretschmann, A.; Seufert, K.; Auwärter, W.; Barth, J. V.; Görling, A.; Steinrück, H.-P.; Gottfried, J. M. The Surface Trans Effect: Influence of Axial Ligands on the Surface Chemical Bonds of Adsorbed Metalloporphyrins. J. Am. Chem. Soc. 2011, 133, 6206-6222.

(6) Scheybal, A.; Ramsvik, T.; Bertschinger, R.; Putero, M.; Nolting, F.; Jung, T. A. Induced Magnetic Ordering in a Molecular Monolayer. Chem. Phys. Lett. 2005, 411, 214-220.

(7) Wende, H.; Bernien, M.; Luo, J.; Sorg, C.; Ponpandian, N.; Kurde, J.; Miguel, J.; Piantek, M.; Xu, X.; Eckhold, P.; Kuch, W.; Baberschke, K.; Panchmatia, P. M.; Sanyal, B.; Oppeneer, P. M.; Eriksson, O. Substrate-Induced Magnetic Ordering and Switching of Iron Porphyrin Molecules. Nat. Mater. 2007, 6, 516-520.

(8) Bernien, M.; Xu, X.; Miguel, J.; Piantek, M.; Eckhold, P.; Luo, J.; Kurde, J.; Kuch, W.; Baberschke, K.; Wende, H.; Srivastava, P. FePorphyrin Monolayers on Ferromagnetic Substrates: Electronic Structure and Magnetic Coupling Strength. Phys. Rev. B 2007, 76, 214406.

(9) Bernien, M.; Miguel, J.; Weis, C.; Ali, M. E.; Kurde, J.; Krumme, B.; Panchmatia, P. M.; Sanyal, B.; Piantek, M.; Srivastava, P.; Baberschke, K.; Oppeneer, P. M.; Eriksson, O.; Kuch, W.; Wende, $\mathrm{H}$. Tailoring the Nature of Magnetic Coupling of Fe-Porphyrin Molecules to Ferromagnetic Substrates. Phys. Rev. Lett. 2009, 102, 047202.

(10) Chylarecka, D.; Wäckerlin, C.; Kim, T. K.; Müller, K.; Nolting, F.; Kleibert, A.; Ballav, N.; Jung, T. A. Self-Assembly and Superexchange Coupling of Magnetic Molecules on Oxygen-Reconstructed Ferromagnetic Thin Film. J. Phys. Chem. Lett. 2010, 1, 1408-1413.

(11) Chylarecka, D.; Kim, T. K.; Tarafder, K.; Müller, K.; Gödel, K.; Czekaj, I.; Wäckerlin, C.; Cinchetti, M.; Ali, M. E.; Piamonteze, C.; Schmitt, F.; Wüstenberg, J.-P.; Ziegler, C.; Nolting, F.; Aeschlimann, M.; Oppeneer, P. M.; Ballav, N.; Jung, T. A. Indirect Magnetic Coupling of Manganese Porphyrin to a Ferromagnetic Cobalt Substrate. J. Phys. Chem. C 2011, 115, 1295-1301.

(12) Iacovita, C.; Rastei, M.; Heinrich, B.; Brumme, T.; Kortus, J.; Limot, L.; Bucher, J. Visualizing the Spin of Individual CobaltPhthalocyanine Molecules. Phys. Rev. Lett. 2008, 101, 116602.

(13) Brede, J.; Atodiresei, N.; Kuck, S.; Lazić, P.; Caciuc, V.; Morikawa, Y.; Hoffmann, G.; Blügel, S.; Wiesendanger, R. Spin- and Energy-Dependent Tunneling through a Single Molecule with Intramolecular Spatial Resolution. Phys. Rev. Lett. 2010, 105, 047204.

(14) Javaid, S.; Bowen, M.; Boukari, S.; Joly, L.; Beaufrand, J.-B.; Chen, X.; Dappe, Y.; Scheurer, F.; Kappler, J.-P.; Arabski, J.; Wulfhekel, W.; Alouani, M.; Beaurepaire, E. Impact on Interface Spin Polarization of Molecular Bonding to Metallic Surfaces. Phys. Rev. Lett. 2010, 105, 077201.

(15) Lach, S.; Altenhof, A.; Tarafder, K.; Schmitt, F.; Ali, M. E.; Vogel, M.; Sauther, J.; Oppeneer, P. M.; Ziegler, C. Metal-Organic Hybrid Interface States of A Ferromagnet/Organic Semiconductor Hybrid Junction As Basis for Engineering Spin Injection in Organic Spintronics. Adv. Funct. Mater. 2012, 22, 989-997.

(16) Wäckerlin, C.; Chylarecka, D.; Kleibert, A.; Müller, K.; Iacovita, C.; Nolting, F.; Jung, T. A.; Ballav, N. Controlling Spins in Adsorbed Molecules by a Chemical Switch. Nat. Commun. 2010, 1, 61.

(17) Wäckerlin, C.; Tarafder, K.; Siewert, D.; Girovsky, J.; Hählen, T.; Iacovita, C.; Kleibert, A.; Nolting, F.; Jung, T. A.; Oppeneer, P. M.; Ballav, N. On-Surface Coordination Chemistry of Planar Molecular Spin Systems: Novel Magnetochemical Effects Induced by Axial Ligands. Chem. Sci. 2012, 3, 3154-3160.

(18) Sanvito, S. Molecular Spintronics. Chem. Soc. Rev. 2011, 40, 3336. 
(19) Stöhr, J.; Siegmann, H. C. Magnetism: From Fundamentals to Nanoscale Dynamics, 1st ed.; Springer: Berlin, Germany, 2006.

(20) Gopakumar, T. G.; Matino, F.; Naggert, H.; Bannwarth, A.; Tuczek, F.; Berndt, R. Electron-Induced Spin Crossover of Single Molecules in a Bilayer on Gold. Angew. Chem., Int. Ed. 2012, 51, 6262-6266.

(21) Miyamachi, T.; Gruber, M.; Davesne, V.; Bowen, M.; Boukari, S.; Joly, L.; Scheurer, F.; Rogez, G.; Yamada, T. K.; Ohresser, P.; Beaurepaire, E.; Wulfhekel, W. Robust Spin Crossover and Memristance Across a Single Molecule. Nat. Commun. 2012, 3, 938.

(22) Gopakumar, T. G.; Tang, H.; Morillo, J.; Berndt, R. Transfer of $\mathrm{Cl}$ Ligands between Adsorbed Iron Tetraphenylporphyrin Molecules. J. Am. Chem. Soc. 2012, 134, 11844-11847.

(23) Cinchetti, M.; Heimer, K.; Wüstenberg, J.-P.; Andreyev, O.; Bauer, M.; Lach, S.; Ziegler, C.; Gao, Y.; Aeschlimann, M. Determination of Spin Injection and Transport in a Ferromagnet/ Organic Semiconductor Heterojunction by Two-Photon Photoemission. Nat. Mater. 2008, 8, 115-119.

(24) Isvoranu, C.; Wang, B.; Schulte, K.; Ataman, E.; Knudsen, J.; Andersen, J. N.; Bocquet, M. L.; Schnadt, J. Tuning the Spin State of Iron Phthalocyanine by Ligand Adsorption. J. Phys.: Condens. Matter 2010, 22, 472002.

(25) Isvoranu, C.; Wang, B.; Ataman, E.; Knudsen, J.; Schulte, K.; Andersen, J. N.; Bocquet, M.-L.; Schnadt, J. Comparison of the Carbonyl and Nitrosyl Complexes Formed by Adsorption of $\mathrm{CO}$ and $\mathrm{NO}$ on Monolayers of Iron Phthalocyanine on $\mathrm{Au}(111)$. J. Phys. Chem. C 2011, 115, 24718-24727.

(26) Ali, M. E.; Sanyal, B.; Oppeneer, P. M. Tuning the Magnetic Interaction between Manganese Porphyrins and Ferromagnetic Co Substrate through Dedicated Control of the Adsorption. J. Phys. Chem. C 2009, 113, 14381-14383.

(27) Wäckerlin, C.; Kartick, T.; Girovsky, J.; Nowakowski, J.; Hählen, T.; Shchyrba, A.; Siewert, D.; Kleibert, A.; Nolting, F.; Oppeneer, P. M.; Jung, T. A.; Ballav, N. Ammonia Coordination Introducing a Magnetic Moment in On-Surface Low-Spin Porphyrin. Angew. Chem., Int. Ed. 2013, 52, 4568-4571.

(28) Miguel, J.; Hermanns, C. F.; Bernien, M.; Krüger, A.; Kuch, W. Reversible Manipulation of the Magnetic Coupling of Single Molecular Spins in Fe-Porphyrins to a Ferromagnetic Substrate. J. Phys. Chem. Lett. 2011, 2, 1455-1459.

(29) Loth, S.; Baumann, S.; Lutz, C. P.; Eigler, D. M.; Heinrich, A. J. Bistability in Atomic-Scale Antiferromagnets. Science 2012, 335, 196199.

(30) Khajetoorians, A. A.; Wiebe, J.; Chilian, B.; Wiesendanger, R. Realizing All-Spin-Based Logic Operations Atom by Atom. Science 2011, 332, 1062-1064.

(31) Wäckerlin, C.; Nowakowski, J.; Liu, S.-X.; Jaggi, M.; Siewert, D.; Girovsky, J.; Shchyrba, A.; Hählen, T.; Kleibert, A.; Oppeneer, P. M.; Nolting, F.; Decurtins, S.; Jung, T. A.; Ballav, N. Two-Dimensional Supramolecular Electron Spin Arrays. Adv. Mater. 2013, 25, 24042408.

(32) Gambardella, P.; Stepanow, S.; Dmitriev, A.; Honolka, J.; de Groot, F. M. F.; Lingenfelder, M.; Gupta, S. S.; Sarma, D. D.; Bencok, P.; Stanescu, S.; Clair, S.; Pons, S.; Lin, N.; Seitsonen, A. P.; Brune, H.; Barth, J. V.; Kern, K. Supramolecular Control of the Magnetic Anisotropy in Two-Dimensional High-Spin Fe Arrays at a Metal Interface. Nat. Mater. 2009, 8, 189-193.

(33) Umbach, T. R.; Bernien, M.; Hermanns, C. F.; Krüger, A.; Sessi, V.; Fernandez-Torrente, I.; Stoll, P.; Pascual, J. I.; Franke, K. J.; Kuch, W. Ferromagnetic Coupling of Mononuclear Fe Centers in a SelfAssembled Metal-Organic Network on $\mathrm{Au}(111)$. Phys. Rev. Lett. 2012, 109, 267207.

(34) Stróżecka, A.; Soriano, M.; Pascual, J. I.; Palacios, J. J. Reversible Change of the Spin State in a Manganese Phthalocyanine by Coordination of CO Molecule. Phys. Rev. Lett. 2012, 109, 147202.

(35) Krull, C.; Robles, R.; Mugarza, A.; Gambardella, P. Site- and Orbital-Dependent Charge Donation and Spin Manipulation in Electron-Doped Metal Phthalocyanines. Nat. Mater. 2013, 12, 337343.
(36) Liu, L.; Yang, K.; Jiang, Y.; Song, B.; Xiao, W.; Li, L.; Zhou, H.; Wang, Y.; Du, S.; Ouyang, M.; Hofer, W. A.; Castro Neto, A. H.; Gao, H.-J. Reversible Single Spin Control of Individual Magnetic Molecule by Hydrogen Atom Adsorption. Sci. Rep. 2013, 3, 1210. 